\title{
AVANCES DE LA CONTAMINACIÓN AMBIENTAL EN LAS AGUAS DEL GOLFO DE MORROSQUILLO
}

\section{ADVANCES IN ENVIRONMENTAL POLLUTION IN THE WATERS OF THE MORROSQUILLO GULF}

\author{
MONTESINO TORRES, OSWALDO ENRIQUE ${ }^{* *}$ Ing.Civil, GUTIÉRREZ RIBON, GUILLERMO1 M.Sc, MONROY PINEDA, \\ MARÍA CECILIA ${ }^{1}$ M.Sc.
}

${ }^{1}$ Universidad de Sucre, Facultad de Ingeniería, Programa de Ingeniería Civil. Maestría en Ciencias Ambientales Universidad de Sucre - SUE Caribe.

\section{Palabras Clave:}

Acuífero,

Sobreexplotación,

Salinización,

Contaminantes,

Intrusión marina,

ICAM.

Key words:

Aquifer,

Overexploitation,

Salinization,

Pollution,

Seawater

Intrusion,

ICAM.

\section{Resumen}

Las aguas del Golfo de Morrosquillo están contaminadas respectivamente, el mar, por vertimientos de materia residual, hidrocarburos y solidos suspendidos, y las aguas del acuífero, por salinización, dureza y contaminación bacterial. Las concentraciones presentes, entre otras, de hidrocarburos HDD en sus aguas marinas son inferiores al valor de referencia $(<10 \mu \mathrm{g} / \mathrm{L})$ para aguas no contaminadas. Se observa tendencia al decrecimiento, con excepción de los casos puntuales en caño Pechelin, caño Francés, Coveñas - Coquerita y arroyo Villeros, ocasionado por manejo de crudo y sus derivados, operaciones de transporte y actividades marítimas propias de la región. El Programa de Monitoreo de la Calidad de las Aguas Marinas y Costeras de Colombia - REDCAM, nació en el año 2001, como la estrategia nacional para cumplir con el objetivo de Evaluar los efectos de actividades humanas sobre los ecosistemas marinos y costeros. Desde entonces, la REDCAM ha servido de soporte al Sistema de Información Ambiental para Colombia - SIAC y es parte integral del Sistema de Información del Recurso Hídrico Marino - SIRH (MADS, Decreto 1323/2007). Este trabajo es un estudio de caso del avance del deterioro de las aguas marinas en el Golfo sucreño, y se hace con muestreo de aguas en 31 estaciones. Sin embargo, cuando se trata de evaluar la contaminación marina hay tres matrices que deben ser objeto de estudio: agua, sedimento y biota (Lucía Viñas. Ministerio de Agricultura, Alimentación y Medio Ambiente (MAAMA, 2012).
INFORMACIÓN

Recibido: 17-10-2013;

Aceptado: $10-12-2014$. Correspondencia autor: oswaldomontesino@gmail.com

\begin{abstract}
The Gulf waters are polluted Morrosquillo respectively the sea by dumping of waste matter, hydrocarbons and suspended solids, and the waters of the aquifer, salinity, hardness and bacterial contamination. The concentrations present, among others, hydrocarbon HDD in their marine waters are lower than the reference value $(<10 \mathrm{ug} / \mathrm{L})$ for unpolluted waters. Decreasing trend is observed, with the exception of specific cases Pechelin spout, spout French, Coveñas - Coquerita and Villeros stream, caused by handling of crude oil and its derivatives, own transport operations and maritime activities in the region. Monitoring Program Quality and Coastal Marine Waters of Colombia - REDCAM, born in 2001, as the national strategy to meet the objective of evaluate the effects of human activities on marine and coastal ecosystems. Since then, has served REDCAM Support Environmental Information System for Colombia - SIAC and is an integral part of the Information System on Water Resources Marino - HRIS (MADS, Decree 1323/2007). This paper is a case study of the further deterioration in the marine waters in the Gulf sucreño, and is made with water sampling at 31 stations. However, when it comes to assess marine pollution are three matrices that must be studied: water, sediment and biota (Viñas Lucia, Ministry of Agriculture, Food and Environment (MAAMA, 2012).
\end{abstract}




\section{Introducción}

Definición y Modos de Contaminación: Contaminación, en general, como concepto, trata de la presencia de materia o energía cuya naturaleza, ubicación o cantidad produce efectos indeseables $u$ objetables sobre los ecosistemas. En otros términos, es la alteración hecha por el hombre o inducida por el hombre a la integridad física, biológica, química y radiológica del medio ambiente (MADS, RAS, 2000).

El deterioro de la calidad del agua, marina ó subterránea, puede deberse a causas naturales o a la actividad humana. En general, al hablar de contaminación se hace referencia a esta última, por ejemplo, un vertido industrial. En muchas ocasiones, la distinción no es fácil, pues una actividad humana no contaminante (los bombeos, en general) puede alterar un equilibrio previo, provocando el deterioro de la calidad del agua subterránea (SÁNCHEZ, 2009).

Las vías por las que distintas sustancias llegan a los acuíferos contaminando las aguas subterráneas son muy diversas (SÁNCHEZ, 2009).

Infiltración de sustancias depositadas en superficie, o de la lluvia a través de ellas.

Filtración de sustancias almacenadas bajo tierra, o disolución de ellas por el agua subterránea.

Filtración desde un río influente

Derrames o rezumes accidentales de depósitos o conducciones, superficiales o enterrados.

Desde la superficie, a través de captaciones abandonadas o mal construidas.

Desde otro acuífero, a través de las captaciones.

Inyección en pozos. En ocasiones ocultamente, otras veces tras un minucioso estudio técnico.

Actividades contaminantes: Las principales actividades humanas que generan contaminación de las aguas subterráneas se pueden englobar en los siguientes grupos: a. Residuos sólidos urbanos, b. Aguas residuales, c. Actividades agrícolas, d. Ganadería, e. Actividades industriales y mineras, $f$. Actividades nucleares (SÁNCHEZ, 2009).

El presente trabajo es un estudio de caso denominado "Avances de la Contaminación Ambiental de las Aguas del Golfo de Morrosquillo " el cuál se ha realizado con base en los resultados tanto de los muestreos de aguas tomadas en las 31 estaciones, como en las respectivas pruebas in situ y en laboratorios. Es un estudio de calidad de aguas marinas pero que se queda allí en el estado líquido, es decir, aún no se complementa en rigor científico con la cobertura de investigación de contaminación en los sedimentos y en los organismos del ecosistema, particularmente los bentónicos.

\section{Materiales y Métodos}

Fase de Campo y Laboratorio: Para la determinación de la calidad del agua marina del golfo de Morrosquillo y de otras regiones del Caribe de Colombia, se tomó la información proveniente de los departamentos de La Guajira, Magdalena, Atlántico, Córdoba, Sucre, Chocó, Cauca y Nariño, correspondientes, a: época lluviosa (agosto - noviembre del 2012), y época de sequía (febrero - junio del 2013). Los muestreos se realizaron en el Instituto de Investigaciones Marinas y Costeras (INVEMAR) con apoyo de funcionarios de las Corporaciones de cada Departamento. Los muestreos de San Andrés, Bolívar, Antioquia y Valle del Cauca fueron realizados directamente por las Corporaciones Autónomas Regionales y de Desarrollo Sostenible. Los análisis de laboratorio se orientaron siguiendo lineamientos del Manual de Técnicas Analíticas de Parámetros Físico-químicos y Contaminantes Marinos (GARAY et al., 2003a), y el Standard Methods (APHA/ AWWA/ WEF. 2012. Apud. REDCAM, 2013).

En el laboratorio de Calidad Ambiental Marina de INVEMAR se hicieron las mediciones de los departamentos de La Guajira, Magdalena, Atlántico, Córdoba, Sucre, Chocó, Cauca y Nariño; los plaguicidas de San Andrés y Bolívar; los hidrocarburos de San Andrés y Antioquia; y los metales pesados de San Andrés. Las demás variables fueron medidas en los laboratorios de las Corporaciones (CORALINA), (CVC), (CARDIQUE) y (CORPOURABÁ), que cuentan con la logística, equipos y profesionales entrenados para hacer los análisis.

En el área del acuífero costero golfo de Morrosquillo (Sucre), el suelo está dedicado a las actividades turísticas y agropecuarias; el agua subterránea está sujeta a la sobreexplotación mediante más de 1.100 pozos con profundidades que oscilan entre dos y $120 \mathrm{~m}$, abasteciendo a una población de 79.080 habitantes, en donde un $40 \%$ corresponde a población flotante o turística, que se concentra en tres períodos vacacionales, de estiaje, que equivalen a un tercio del año hidrológico, localizados en los extremos y en el centro del mismo (MONTESINO, 2014).

Por otra parte, las aguas superficiales del continente en las subregiones Montes de María, Sabanas y Morrosquillo del departamento de Sucre (Fig. 1), entre otros, arroyos Guainí, Pechelín, Villeros, importantes en caudal y recorrido, y el río Sinú en el departamento de Córdoba, afluentes del mar Caribe, se han convertido en canales de vertimientos contaminantes al acuífero costero y al mismo mar por los residuos domésticos municipales y agropecuarios que reciben por actividades antrópicas ante la inexistencia de sistema de tratamiento alguno (MONTESINO, 2014). 


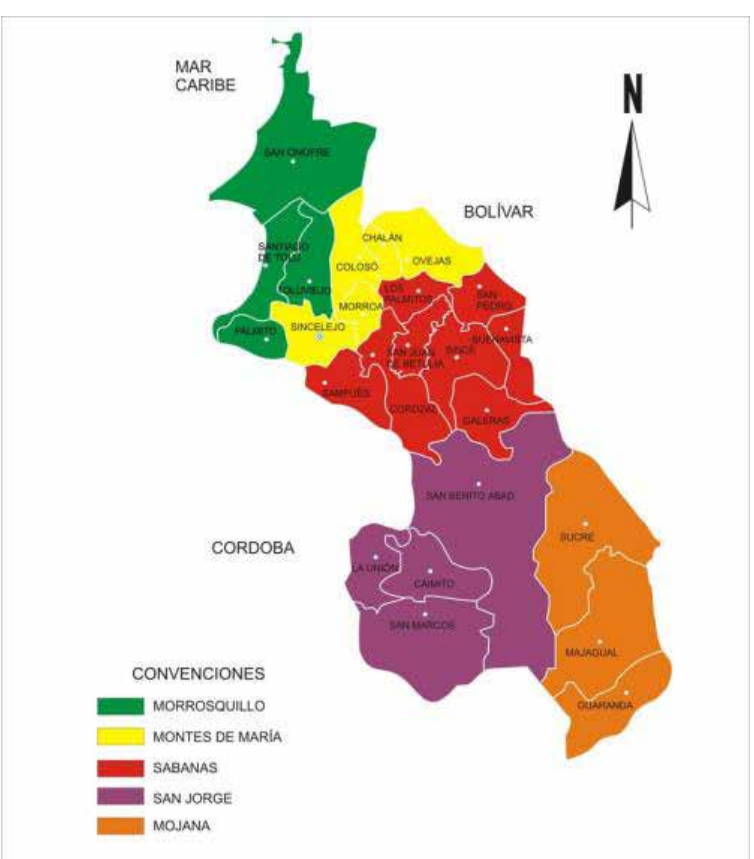

Figura 1. Mapa de Sucre y sus subregiones Fuente: Gobernación de Sucre (2001).

\section{Presentación del caso}

Contaminación de las aguas del golfo de Morrosquillo: La contaminación marina es un registro de huellas de las actividades humanas, y las aguas del golfo de Morrosquillo no son la excepción. Aunque las áreas oceánicas se encuentran relativamente limpias, en las aguas menos profundas, particularmente golfos, bahías y estuarios, se presentan graves conflictos; allí se suman la contaminación de aguas, sedimentos y organismos. Cada vez se ha hecho más claro que las actividades realizadas en la Tierra son las principales fuentes de contaminación de los mares y las costas. Sin embargo, también se reconoce que no solo la contaminación es responsable del deterioro de los mares y océanos, también lo son el daño físico directo a los ecosistemas y la sobreexplotación de los recursos (GESAMP, 2001).

Las aguas del golfo de Morrosquillo, tanto en el ecosistema marino como en el entorno del acuífero costero, están contaminadas respectivamente, el mar, por vertimientos de materia orgánica residual, hidrocarburos y sedimentos, y las aguas del acuífero, por salinización y contaminación bacterial (MONTESINO, 2014).

Antecedentes: Las características del régimen climático e hídrico del área del acuífero (Fig. 2), así como las características físico-químicas de las aguas y

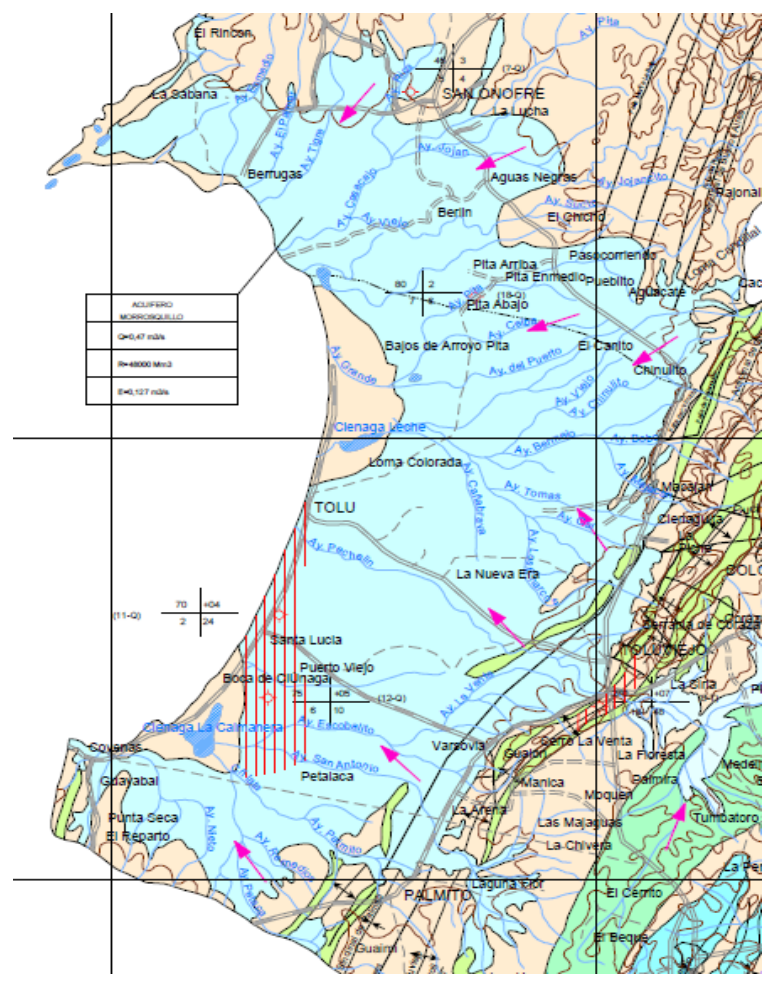

Figura 2. Mapa Hidrogeológico de Sucre con delimitación del acuífero de Morrosquillo. Fuente: (INGEOMINAS, 2002).

el abatimiento de los niveles de los pozos en la época de sequía evidencian, de acuerdo a las investigaciones antecedentes que, existe desde hace 30 años, al menos, un proceso de salinización por intrusión marina que ha venido alterando la calidad del recurso hídrico subterráneo, el cual es fuente de abastecimiento para el consumo humano, con las consecuencias pertinentes, aumento de los costos del sistema de acueducto y del servicio público por la requerida potabilización requerida (MONTESINO, 2014).

Mención específica amerita la alteración de la calidad del agua marina en el golfo de Morrosquillo y del agua subterránea en el acuífero costero de la Subregión del mismo nombre, por su condición de ecosistemas sedes de actividades portuarias, asentamientos humanos, con actividades turísticas y hoteleras, el uno, y sujeto al uso indebido de su suelo y a sobreexplotación el otro, y por ende, ambos receptores de residuos y vertimientos de alta peligrosidad como fuentes contaminantes permanentes (MONTESINO, 2014).

Contaminación Marina: El Programa de Monitoreo de la Calidad de las Aguas Marinas y Costeras de Colombia - REDCAM, nació en el año 2001, como la estrategia nacional para cumplir con el objetivo de 
evaluar los efectos de actividades humanas sobre los ecosistemas marinos y costeros, con miras a disponer en forma permanente de un diagnóstico actualizado de las condiciones ambientales, planteado en el PNICM -Programa Nacional de Investigación, evaluación, prevención, reducción y control de las fuentes marinas y terrestres de contaminación al mar". Desde entonces, la REDCAM ha servido de soporte al Sistema de Información Ambiental para Colombia - SIAC y es parte integral del Sistema de Información del Recurso Hídrico Marino - SIRH (MADS, Decreto 1323/2007).

En España, el Instituto de Oceanografía realiza las actividades de seguimiento ambiental marino; citándolo a través de Lucía Viñas en MAAMA (2012) se tiene por cierto que "la mayoría de los contaminantes se encuentran en el agua de mar en cantidades muy pequeñas por lo que su cuantificación analítica es compleja y costosa. Además, las concentraciones en el agua, especialmente en zonas costeras, son variables con el tiempo ya que dependen de las mareas, las corrientes, los vientos ó los vertidos intermitentes. Por otra parte, la información que proporciona el análisis en el agua refleja solamente la contaminación existente en el momento de la toma de muestras que puede haber variado a las pocas horas ó a los pocos días." Por otra parte," los sedimentos son preferibles a las muestras de agua como matriz para el seguimiento de la calidad ambiental, ya que las concentraciones en los mismos son mucho mayores y manos variables en el tiempo y en el espacio, reflejando de modo integrado el estado de contaminación de una zona." y en cuanto a la matriz de organismos, se ha establecido que en relación con la biota, "cuando una sustancia no puede ser metabolizada ó se metaboliza de forma lenta, tenderá a acumularse en los tejidos de los seres vivos alcanzando concentraciones superiores a las que encontramos en el ambiente. En el caso particular de los organismos acuáticos, la fuente de tóxico suele ser el agua. En éste caso, se define como factor de bioconcentración (FBC) a la concentración alcanzada en el organismo. Muchos organismos marinos acumulan contaminantes en sus tejidos a niveles muy superiores a los presentes en el agua que los rodea, sin que existan efectos tóxicos aparentes. Esta característica ofrece ventajas evidentes para el desarrollo de los programas de seguimiento de la contaminación marina" (MAAMA, 2012).

Contaminación del Acuífero Costero Morrosquillo: Desde el año 1997, mediante los resultados de trabajos de investigación se ha establecido la contaminación por salinización, entendida como el aumento de la salinidad, de las aguas subterráneas del acuífero costero Morrosquillo. Los antecedentes se describen a través de los dos escenarios siguientes:

Balance Hidrogeológico. Mediante el Análisis de la relación de extracciones vs. recargas, en el campo de pozos del acuífero, y en el marco del balance hidrogeológico, se estableció que la operación de abastecimiento se hace enmarcada en la sobreexplotación, desde el año 2000, al menos, como lo estableció GUTIÉRREZ (2004); éste resultado tiene su mayor incidencia en el fenómeno de la invasión marina y su impacto ambiental.

La demanda anual de agua para la población del área de estudio fue calculada con base en las disposiciones de dotación per cápita y nivel de complejidad establecidos en el REGLAMENTO TÉCNICO DE AGUA POTABLE Y SANEAMIENTO BÁSICO RAS 2000 (MADS, 2000), vigente en Colombia. Es un volumen cuyo valor ascendió a 1,33 $\mathrm{Hm}^{3}$ /año, para el 2002. En contraste, la extracción total anual de agua del acuífero, en el área de estudio, se calculó en $6,43 \mathrm{Hm}^{3}$ /año.

Se determinó una diferencia de $5.0 \mathrm{Hm}^{3}$ /año entre los dos eventos, volumen que califica como una explotación desmedida del acuífero y que tipifica un uso indebido del recurso hídrico, a lo largo de cada período anual; es un factor de sobreexplotación (GUTIÉRREZ, 2004).

Relación Acuífero - Mar. Se detalla ésta relación en torno al balance hídrico, expresándolo conforme lo hacen autores como BINDEMAN et al. (1976) y RODRÍGUEZ (2001) (Apud. PÉREZ, 1995).

Entradas $=$ Salidas $+\mathrm{dW}$

En donde dW es la variación de las reservas.

Asignando los valores característicos para la cuenca en estudio se tiene (Tabla 1):

Tabla 1. Resumen Balance Hídrico. Fuente: GUTIERREZ (2004)

\begin{tabular}{|c|c|c|c|}
\hline \multicolumn{2}{|l|}{ Entradas (Hm³/año) } & \multicolumn{2}{|l|}{ 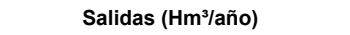 } \\
\hline - Infiltración Eficaz : & 30.261 & $\begin{array}{l}\text { Evapotranspiración Real: } \\
\text { (ETR) }\end{array}$ & 137.568 \\
\hline $\begin{array}{l}\text {-Recarga Natural } \\
\text { (arroyo Pechelín) }\end{array}$ & 18.940 & Descarga Natural & 0.000 \\
\hline $\begin{array}{l}\text {-Recarga Natural Inducida } \\
\text { (mar local) }\end{array}$ & 19.155 & Salida de agua Subterránea & 0.000 \\
\hline Entrada de Agua Subterránea & 0.000 & $\begin{array}{l}\text { Descarga Artificial } \\
\text { (extracciones) }\end{array}$ & 6.426 \\
\hline Recarga Artificial & 0.899 & & \\
\hline Sumatoria & 69.255 & Sumatoria & 143.994 \\
\hline
\end{tabular}

Por lo tanto dW, la variación de las reservas tiene el valor de $75,0 \mathrm{Hm}^{3}$ /año, aproximadamente, de carácter negativo, es decir, es una disminución de las mismas.

Con base en lo anterior, la variación de las reservas del acuífero en el período del análisis hidrológico (30 
años hasta el 2004) ha alcanzado el volumen de 2.250 $\mathrm{Hm}^{3}$. Por su parte el volumen de agua de mar que ha invadido el acuífero es de $575,0 \mathrm{Hm}^{3}$ aproximadamente, en el mismo período.

De la información general del mapa hidrogeológico de Sucre (INGEOMINAS, 2002) se conoce que los recursos del acuífero Morrosquillo han sido estimadas en $48.000 \mathrm{Hm}^{3}$. En ésta investigación se asumen esas reservas como reservas permanentes de los estratos acuíferos, incluido el acuífero libre. Para acuíferos confinados éstas reservas equivalen a la reserva total $\left(\mathrm{W}_{t}\right)$ ó reserva máxima media, debido a que la variación de las reservas (dW) es muy pequeña en éste tipo de acuíferos. En el caso que nos ocupa, dW equivale al $4,7 \%$ de la reserva total.

Como parte del análisis, asumida la definición de tasa de renovación como la relación entre la variación media de la reserva y las reservas totales del acuífero (GUTIERREZ, 2004), ésta tasa se calcula como:

\section{Tasa de Renovación=75/48000=0,0015625/año}

Análogamente, la definición de Período de Renovación es el tiempo que sería necesario para reconstruir las reservas totales, si se han agotado a través del volumen reemplazado anualmente. Es decir, es el inverso de la Tasa de Renovación, y por ende, su valor es 640 años (PÉREZ, 1995).

Elementos de la contaminación de las aguas, marina y subterránea. En las aguas del mar en el golfo de Morrosquillo se obtuvieron los siguientes valores en las concentraciones de los parámetros in situ y variables físico químicas, temperatura, salinidad, $\mathrm{Ph}$, oxígeno disuelto, solidos suspendidos totales, coliformes totales y termotolerantes, valores que se describen y expresan mediante gráficas.

La primera zona de muestreo es la zona Norte, conformada por aguas marinas que incluyen isla Palma y el islote Santa Cruz (islote artificial), que se encuentran rodeadas de áreas coralinas, ciénagas y manglares, estas se han convertido en zonas turísticas y muestran una alta diversidad de especies, mientras que la zona Golfo afuera tiene características hidrológicas propias de agua superficial ecuatorial tropical, donde se desarrollan zonas de mangle (REDCAM, 2013).En la zona de Tolú predominan extensas playas protegidas por una serie de espolones principalmente de caños y playas, algunas se encuentran limitando pantanos de manglar y llanuras costeras y otras están comunicadas con las bocas de algunos arroyos y ciénagas; además conforman los cordones litorales que individualizan lagunas interiores (REDCAM, 2013) (Fig. 3).

Variables Físico Químicas in situ. La temperatura en las estaciones ubicadas en la zona Norte es de especial interés debido a la presencia de formaciones coralinas y praderas de fanerógamas. En la época lluviosa 2012 el promedio de la temperatura en esta zona fue de $30,5 \pm 0,5^{\circ} \mathrm{C}$ y en época seca de $30,4 \pm$ $0,7{ }^{\circ} \mathrm{C}$, igualmente, los datos históricos (2001-2013) muestran que la temperatura media es $30,3 \pm 0,5^{\circ} \mathrm{C}$ en época seca y de $30,7 \pm 1,0^{\circ} \mathrm{C}$; lo cual da evidencia que en algunas épocas del año los valores están muy cercanos a $30{ }^{\circ} \mathrm{C}$, temperatura asociada a efectos de degradación de corales por blanqueamiento ( GRAHAM

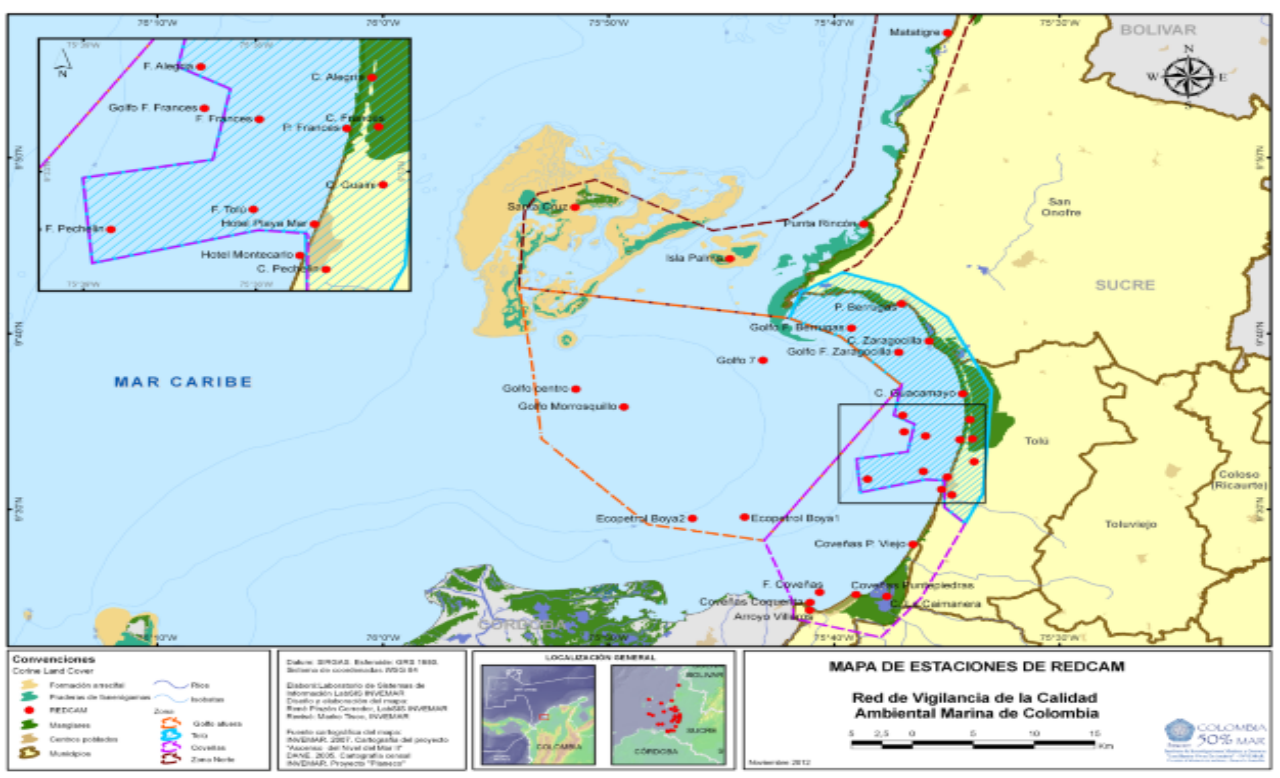

Figura 3. Estaciones y zonas de muestreo de la REDCAM en el departamento de Sucre. Fuente:(REDCAM (2013). 
et al., 2008; KLEYPAS y HOEGHGULDBERG, 2008. Apud. REDCAM, 2013) (Figura 4).

El comportamiento de la salinidad durante el periodo de análisis es congruente con el registrado históricamente, no se observaron grandes variaciones en los valores entre épocas para las estaciones marinas con excepción de Santa Cruz donde la salinidad aumentó 10,2 unidades en época seca respecto a la época lluviosa. En el caso de las estaciones de carácter estuarino y fluvial, donde el proceso de mezcla disminuyó la salinidad especialmente en época de lluvias, las estaciones que presentaron una variación notable entre épocas fueron caño Francés, caño Zaragocilla y caño La Caimanera, lo que evidencia como los factores hidrodinámicos, el régimen de mareas y las descargas de los diferentes efluentes continentales influyen en el comportamiento espacial y temporal de la salinidad en las aguas costeras del Departamento (Fig. 5).
En el periodo actual de análisis los valores de $\mathrm{pH}$ de las aguas superficiales del departamento de Sucre no presentaron variaciones significativas entre las dos épocas. Así, en la época lluviosa 2012 el promedio fue $8,2 \pm 0,1$ y en época lluviosa fue de $8,3 \pm 0,1$; en el caso de las aguas estuarinas y fluviales los valores promedio fueron $7,7 \pm 0,4$ y $8,2 \pm 0,4$ en época lluviosa y seca respectivamente. En la mayoría de estaciones los valores de $\mathrm{pH}$ se ubicaron dentro de los criterios de calidad permisibles de 6,5 a 8,5 y 4.5 a 9.0 para aguas marinas y fluviales respectivamente, establecidos en el Decreto 1594 de 1984 para preservación de fauna y flora (MINSALUD, 1984); a excepción de las estaciones caño Guaini $(8,64)$, arroyo Villeros $(8,82)$ y CoveñasPuntepiedras $(8,52)$ con valores de $\mathrm{pH}$ superiores al límite máximo. Históricamente la tendencia general de la variable es a aumentar en época seca principalmente en las estaciones estuarinas y fluviales como consecuencia de la disminución en los procesos de escorrentía y lixiviados de elementos ácidos en las zonas ribereñas

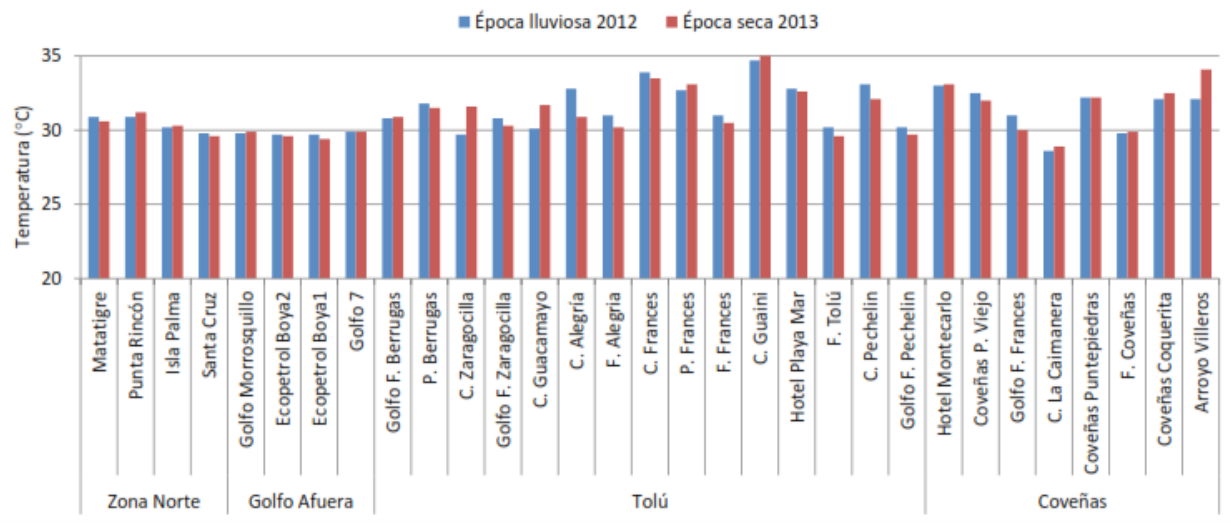

Figura 4. Temperatura $\left({ }^{\circ} \mathrm{C}\right)$ superficial del agua medida en 4 zonas del departamento de Sucre durante la época lluviosa de 2012 y la época seca de 2013 Fuente: REDCAM (2013)

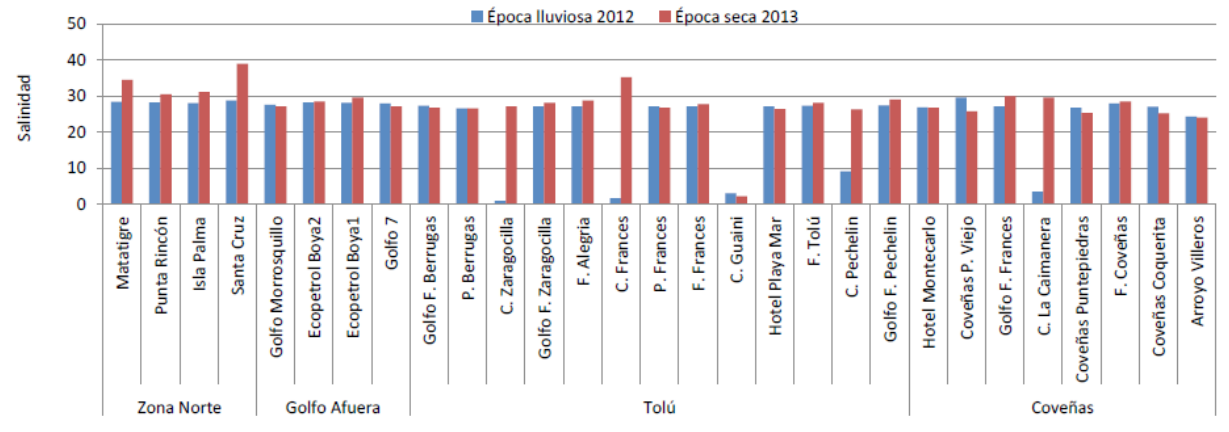

Figura 5. Salinidad del agua superficial en 4 zonas del departamento de sucre medida en la época lluviosa de 2012 y la época seca de 2013. Fuente: REDCAM (2013) 
y la consecuente disminución del $\mathrm{CO} 2$ producido por la oxidación de la materia orgánica y por el aumento de procesos biológicos tales como fotosíntesis y respiración (COGNETTI et al., 2001. (Apud. REDCAM, 2013) (Fig. 6).

El Oxígeno Disuelto (OD) en la mayoría de estaciones estuvo por encima de $4,0 \mathrm{mg} / \mathrm{L}$, considerado el valor mínimo de calidad para la preservación de flora y fauna según la legislación colombiana (MINSALUD, 1984). Las concentraciones fluctuaron entre $4,3 \mathrm{mg} / \mathrm{L}$ y 7,6 $\mathrm{mg} / \mathrm{L}$ (promedio $5,6 \pm 0,7 \mathrm{mg} / \mathrm{L}$ ) en la época lluviosa 2012; mientras que para la época seca 2013, el rango osciló entre 4,6 mg/L y 7,5 mg/L (promedio 5,6 \pm 0,6 $\mathrm{mg} / \mathrm{L}$ ). En las estaciones fluviales y estuarinas los rangos fueron $1,5 \mathrm{mg} / \mathrm{L}$ a $6,1 \mathrm{mg} / \mathrm{L}$ (promedio $4,0 \pm$ $1,5 \mathrm{mg} / \mathrm{L}$ ) para la temporada lluviosa y de $0,1 \mathrm{mg} / \mathrm{L}$ a $15,3 \mathrm{mg} / \mathrm{L}$ (promedio $8,2 \pm 5,1 \mathrm{mg} / \mathrm{L}$ ) para seca. No se observaron variaciones significativas entre épocas $(p>$ 0,05 -Statgraphics $R$ ) para aguas marinas, pero si para aguas estuarinas y fluviales $(p<0,05)$ (Fig. 7).
En las estaciones estuarinas y fluviales de la zona de Tolú, caño Guacamayo (1,54 mg/L), caño Alegría (2,86 $\mathrm{mg} / \mathrm{L})$ y caño Francés $(2,11 \mathrm{mg} / \mathrm{L})$, el OD estuvo por debajo del valor de referencia durante la época lluviosa 2012, mientras que en la época seca 2013 el valor más bajo se registró en caño Alegría $(0,1 \mathrm{mg} / \mathrm{L})$; condición que puede deberse a un incremento en la demanda bioquímica de oxígeno generada por la descarga de material terreo y a las aguas residuales generadas en las áreas que recorren estos caños (VIVAS-AGUAS et al., 2010). Las concentraciones de Sólidos Suspendidos Totales (SST) en las aguas superficiales costeras del departamento de Sucre mostraron amplios intervalos de variación entre épocas y estaciones de muestreo. Los valores fluctuaron entre $1,9 \mathrm{mg} / \mathrm{L}$ y $97,8 \mathrm{mg} / \mathrm{L}$ durante la época de lluvias de 2012 y entre 6,8 mg/L y 179 mg/L durante la época seca de 2013, enmarcándose dentro del rango de valores de SST históricamente medidos en la REDCAM para este departamento (Fig. 8). En términos generales, las concentraciones de SST fueron más altas en los principales caños que desembocan en el mar, en la zona de Tolú como en caño Zaragocilla

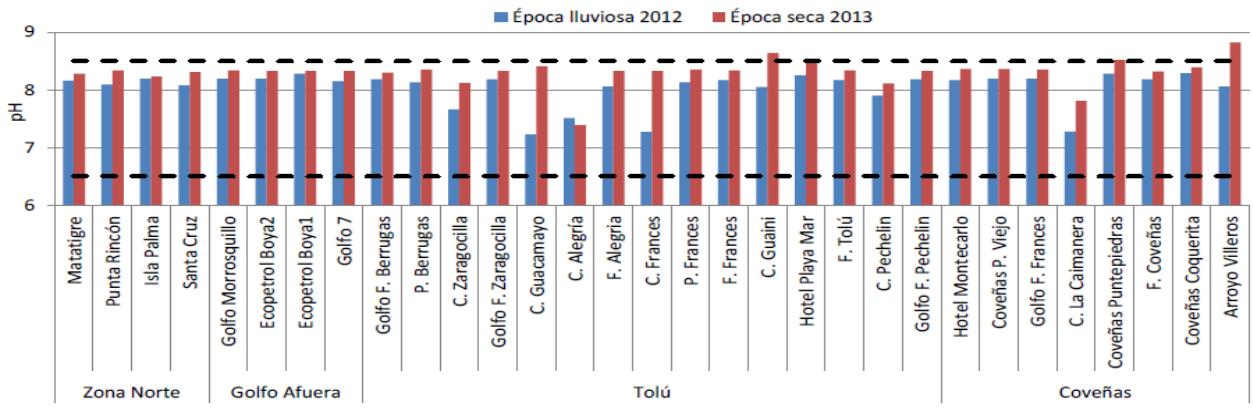

Figura 6. Valores de $\mathrm{pH}$ en aguas superficiales de Sucre medido en la época lluviosa 2012 y la época seca 2013. Las líneas punteadas representan el máximo y mínimo del Rango Permisible (R.P.) para la preservación de flora y fauna en aguas marinas y estuarinas según el Decreto 1594 de 1984. Fuente: MINSALUD (1984).

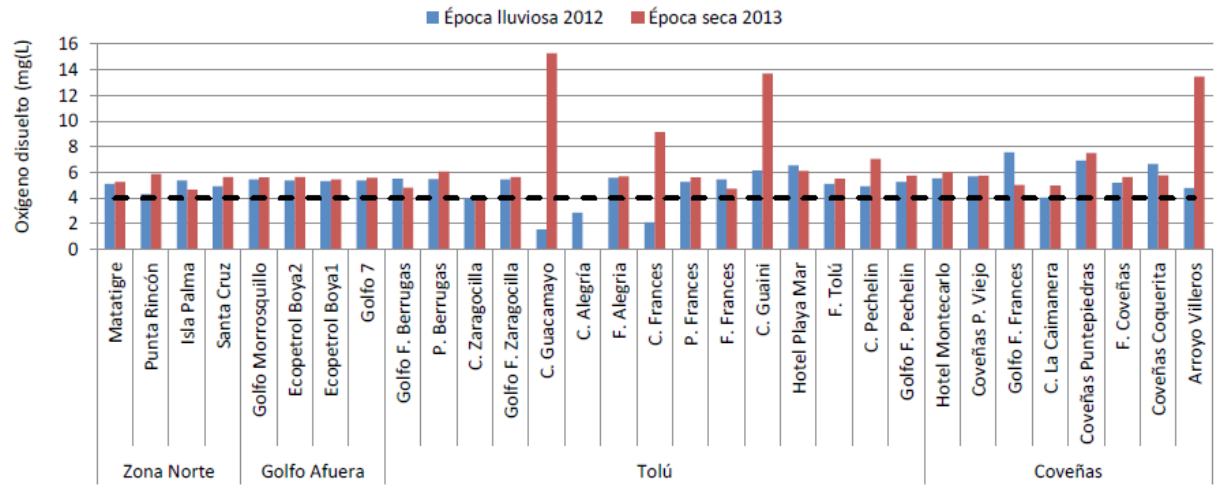

Figura 7.Oxígeno disuelto (mg/L) medido en aguas superficiales del departamento de Sucre, en la época lluviosa 2012 y la época seca 2013. La línea punteada representa el valor Mínimo Permisible (M.P.) para la preservación de flora y fauna según el Decreto 1594. Fuente: (MINSALUD, 1984). 
(147 mg/L), caño Guacamayo (131,0 mg/L), caño Francés (179,0 mg/L) y caño Guaní (72 mg/L) durante la época seca y en hotel Playa Mar (97,8 mg/L) durante la época lluviosa. En el caso de las estaciones marino costeras ubicadas al Norte y en las afueras del Golfo los valores de SST no fueron mayores a $20 \mathrm{mg} / \mathrm{L}$ se observaron pequeños intervalos de variación entre épocas de muestreo. No obstante las concentraciones de sólidos no superaron el promedio histórico de la REDCAM (Fig. 9).

Teniendo en cuenta los efectos adversos que los SST pueden ocasionar en la salud de los ecosistemas coralinos al inhibir los procesos fotosintéticos de las algas asociadas, además de perturbar la reproducción de estos, según los datos de la REDCAM en las estaciones de la zona Norte, caracterizadas por estar rodeadas de extensas áreas coralinas, las concentraciones de SST no estrían afectando la salud de estos ecosistemas, al encontrarse por debajo del valor de referencia (50 mg/L) (FABRICIUS, 2005. Apud. REDCAM, 2013).

Contaminación Microbiológica. La evaluación de la calidad microbiológica de las aguas superficiales del departamento de Sucre se realizó a través de monitoreos semestrales para determinar los indicadores de contaminación fecal, Coliformes Totales (CTT) y Termotolerantes (CTE) en tres zonas costeras del Departamento. Las concentraciones históricas de CTT registradas en la ciénaga la Caimanera, evidenciaron diferencias significativas entre épocas climáticas ( $p<$ $0,05)$, como es el caso de los CTT que en la época lluviosa superaron los límites permisibles para el desarrollo de actividades de contacto secunda pesca, riego de frutas y hortalizas de tallo corto (< 5.000 NMP.100 mL-1) (MINSALUD, 1984) con una concentración promedio de 18,263 \pm 0,84 NMP.100 mL-1; sin embargo, las concentraciones históricas de CTT en la ciénaga la Caimanera se han mantenido por debajo de limites establecido para el desarrollo de actividades de contacto primario natación y buceo (< 1000 NMP.100 mL-1) (MINSALUD, 1984) desde el año 2009, por lo que las altas concentraciones pueden atribuirse a las escorrentías de la temporada lluviosa que arrastran materiales y sedimentos de las actividades realizadas en la superficie de las cuencas urbanas entrando al sistema de forma directa (SUÁREZ et al., 2012. Apud. REDCAM,2013) (Fig. 10).

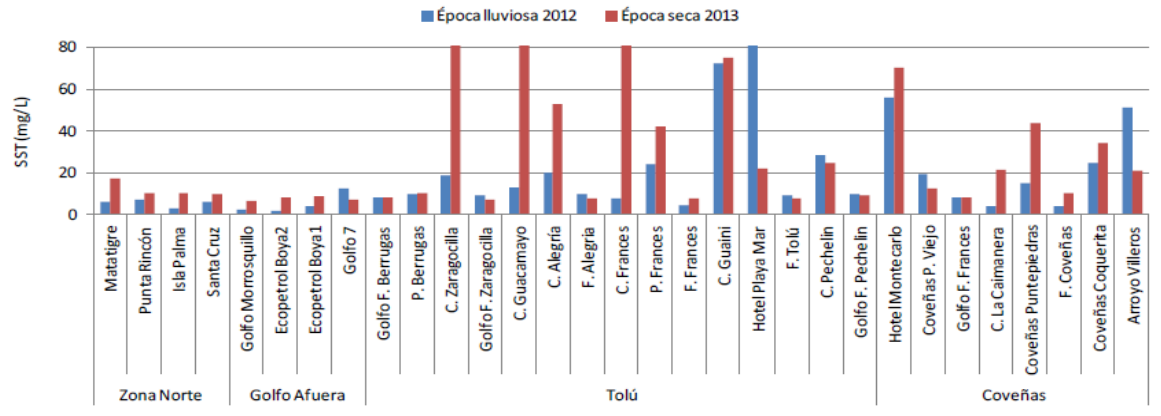

Figura 8. Sólidos suspendidos totales (mg/L) medidos en aguas superficiales del departamento de Sucre en la época lluviosa 2012 y la época seca 2013.

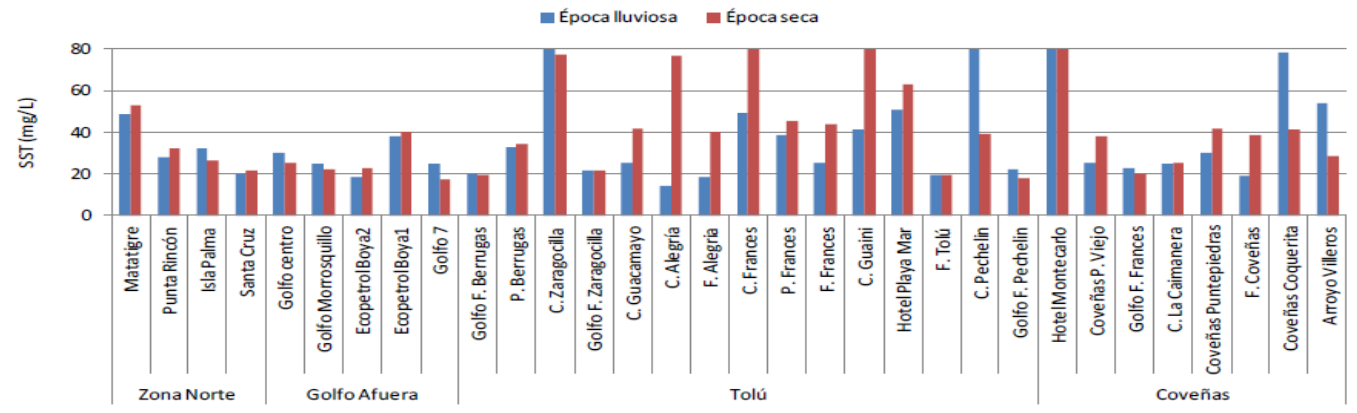

Figura 9. Promedio histórico de Sólidos Suspendidos Totales (mg/L) medidos semestralmente en las épocas lluviosas y secas entre el 2001 y 2013 en aguas superficiales de Sucre 


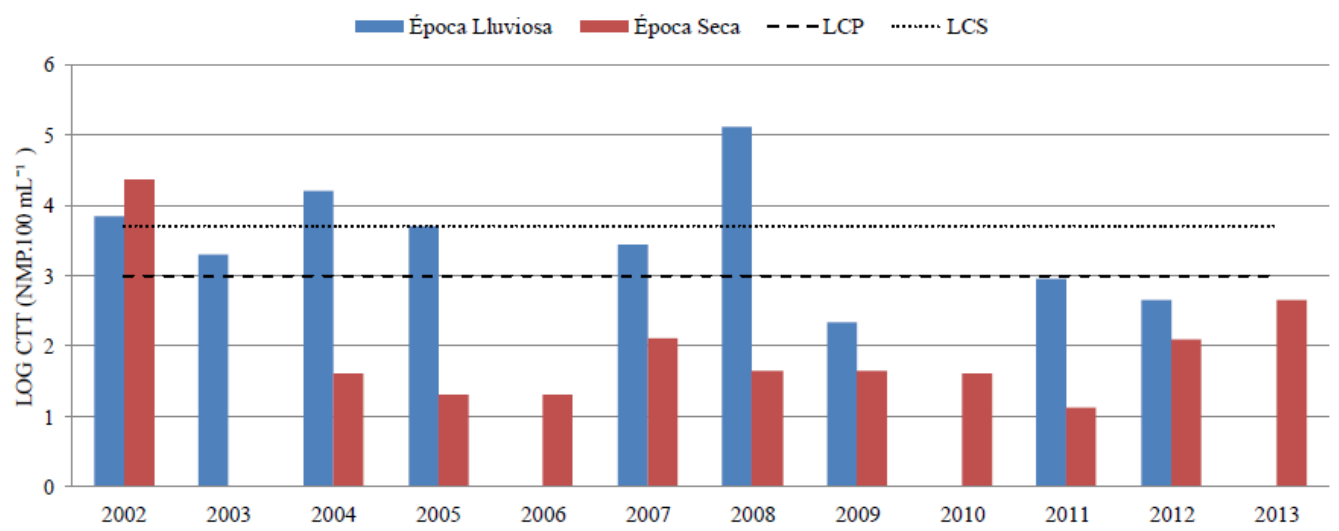

Figura 10. Concentraciones de Coliformes Totales (LOG NMP.100 mL-1) medidas en las estaciones del departamento de Sucre del año 2002- 2013 en la época lluviosa y la época seca. La línea cortada equivale al límite de referencia para aguas destinadas a contacto primario (LCP) (<1000 NMP.100 mL-1) (MINSALUD, 1984) y la línea punteada equivale al límite de referencia para aguas destinadas a contacto secundario (LCS), actividades de pesca, riego de frutas y hortalizas de tallo corto $(<5000$ NMP.100 mL-1). Fuente: MINSALUD, (1984).

La evaluación de la calidad microbiológica de las 10 playas del Departamento se realizó mediante la medición de las concentraciones de CTE. Históricamente, no se evidenciaron diferencias significativas entre épocas climáticas $(p>0,05)$, los casos en que las concentraciones superan el límite establecido para el desarrollo de actividades de contacto primario (< 200 NMP.100 mL-1; MinSalud, 1984) se registraron para ambas épocas, incidiendo Coveñas-Coquerita en un $90 \%$ de las mediciones para la época lluviosa y un $92 \%$ para la época seca (Tabla 2), con concentraciones promedios de $13,037 \pm 0,75$ NMP.100 mL-1 y 2,430 $\pm 0,95$ NMP. 100 mL-1 respectivamente. La calidad de las aguas costeras, se ha considerado un aspecto fundamental para los turistas a la hora de elegir su estancia vacacional (FLORES et al., 2011. Apud. REDCAM, 2013), por lo que una inadecuada calidad puede generar riesgos para la salud de los usuarios (VERGARA et al., 2007. Apud. REDCAM, 2013).

Tabla 2. Concentraciones de Coliformes Termotolerantes (CTE NMP.100 mL-1) medidas en las épocas climáticas lluviosa 2012 y seca 2013. En negrilla se muestran los valores que superan el límite de referencia para aguas destinadas a contacto primario (<200 NMP.100 mL-1). Fuente: MINSALUD (1984).

\begin{tabular}{|c|c|c|c|c|c|c|c|}
\hline \multirow{2}{*}{ Zona } & \multirow{2}{*}{ Playa } & \multicolumn{3}{|c|}{ Época lluviosa 2012} & \multicolumn{3}{|c|}{ Época seca 2013} \\
\hline & & (NMP.100 mL $\left.\mathrm{mL}^{-1}\right)$ & $\%$ casos & $\mathbf{n}^{* *}$ & $\left(\mathrm{NMP} .100 \mathrm{~mL}^{-1}\right)$ & $\%$ casos* & $\mathbf{n}^{* *}$ \\
\hline \multirow{3}{*}{ Norte } & Punta Rincón & 110 & 60 & 10 & 160 & 56 & 9 \\
\hline & Isla Palma & 1,8 & 11 & 9 & 130 & 11 & 9 \\
\hline & Santa Cruz & 490 & 63 & 8 & 410 & 25 & 8 \\
\hline Tolú & P. Berrugas & 18 & 40 & 10 & 210 & 64 & 11 \\
\hline \multirow{4}{*}{ Zona } & Plava & \multicolumn{3}{|c|}{ Época lluviosa 2012} & \multicolumn{3}{|c|}{ Época seca 2013} \\
\hline & Praya & $\left(\mathrm{NMP} .100 \mathrm{~mL}^{-1}\right)$ & $\%$ casos * & $\mathbf{n}^{* *}$ & $\left(\mathrm{NMP} .100 \mathrm{~mL}^{-1}\right)$ & $\%$ casos * & $\mathbf{n}^{* *}$ \\
\hline & P. Frances & 1,8 & 33 & 9 & 360 & 50 & 12 \\
\hline & Hotel Playa Mar & 20 & 70 & 10 & 210 & 73 & 11 \\
\hline \multirow{4}{*}{ Coveñas } & Hotel Montecarlo & 18 & 80 & 10 & 50 & 64 & 11 \\
\hline & $\begin{array}{l}\text { Coveñas P. Viejo } \\
\text { Coveñas }\end{array}$ & 18 & 50 & 10 & 40 & 50 & 10 \\
\hline & Puntepiedras & 4,5 & 40 & 10 & 200 & 67 & 12 \\
\hline & Coveñas Coquerita & 180 & 90 & 10 & 200 & 92 & 12 \\
\hline
\end{tabular}

"*\%de casos de incumplimiento en el análisis histórico con respecto al límite permisible establecido en el decreto 1594 de 1984 para realizar actividades de contacto primario como la natación (CTE <200 NMP.100 mL-1). 
Hidrocarburos. Las concentraciones de Hidrocarburos Disueltos y Dispersos - HDD, en el período de lluvia 2012 y seco 2013 oscilaron en un rango entre 0,07 $\mu \mathrm{g} / \mathrm{L}$ y $2,87 \mu \mathrm{g} / \mathrm{L}$. La tendencia de las concentraciones estuvieron más altas en la época lluviosa de 2012, en las estaciones Matatigre $(1,57 \mu \mathrm{g} / \mathrm{L})$ y Boya 2 Ecopetrol $(1,11 \mu \mathrm{g} / \mathrm{L})$ en la zona Golfo Afuera y en la zona de Coveñas el Golfo Frente al Francés $(2,87 \mu \mathrm{g} / \mathrm{L})$ y playa Berrugas $(1,20 \mu \mathrm{g} / \mathrm{L})$. Por otro lado, en la época seca de 2013 , los valores más altos se registraron en la zona de Coveñas en la estaciones Coveñas Coquerita (1,97 $\mu \mathrm{g} / \mathrm{L})$ y en las playas del hotel Montecarlo $(1,64 \mu \mathrm{g} / \mathrm{L})$. Los residuos de hidrocarburos que se presentan en el Departamento se deben a la actividad portuaria de transporte de petróleo, transporte marítimo, turismo y aguas servidas de los municipios de Tolú y Coveñas; sin embargo, las concentraciones observadas en las estaciones de este Departamento no superan el valor de referencia de $10 \mu \mathrm{g} / \mathrm{L}$ para aguas no contaminadas (UNESCO, 1984. Apud. REDCAM, 2013) (Fig. 11).
El análisis temporal muestra diferencias significativas $(p>0,05)$, registrando las concentraciones promedio más altas en la época lluviosa en las estación caño Francés $(44,48 \pm 130,48 \mu \mathrm{g} / \mathrm{L})$ de la zona Tolú y de la zona Coveñas las estaciones playa Coquerita $(20,75 \pm 33,85 \mu \mathrm{g} / \mathrm{L})$ y arrollo Viñeros $(12,96 \pm 17,56 \mu \mathrm{g} / \mathrm{L})$. Esto se debe principalmente $a$ las escorrentías de aguas de lluvia, transporte de petróleo y marítimo, vertimientos de aguas residuales domésticas y a la actividad turística (Fig. 12).

La variación interanual 2001 a 2013 mostró diferencias $(p<0,05)$, registrando concentraciones de HDD superiores al valor de referencia en los años 2009 en caño Pechelin $(38,17 \mu \mathrm{g} / \mathrm{L})$, en el año 2010 en los caños Francés $(392,41$ $\mu \mathrm{g} / \mathrm{L})$ y Pechelin $(15,04 \mu \mathrm{g} / \mathrm{L})$, las boyas de Ecopetrol TLU1 $(45,58 \mu \mathrm{g} / \mathrm{L})$ y TLU2 $(221,82 \mu \mathrm{g} / \mathrm{L})$, Coveñas Coquerita $(59,82 \mu \mathrm{g} / \mathrm{L})$, golfo frente a Berrugas $(17,01 \mu \mathrm{g} / \mathrm{L})$ y Golfo $7(13,53 \mu \mathrm{g} / \mathrm{L})$ y en 2011 en los caños Zaragocilla (193,0 $\mu \mathrm{g} / \mathrm{L})$, Pechelin $(188,0 \mu \mathrm{g} / \mathrm{L})$ y Francés $(52,7 \mu \mathrm{g} / \mathrm{L})$, los Playas de Coquerita $(16,5 \mu \mathrm{g} / \mathrm{L})$ y frente a los hoteles Montecarlo $(30,1 \mu \mathrm{g} / \mathrm{L})$ y Playa Mar $(17,1 \mu \mathrm{g} / \mathrm{L})$.

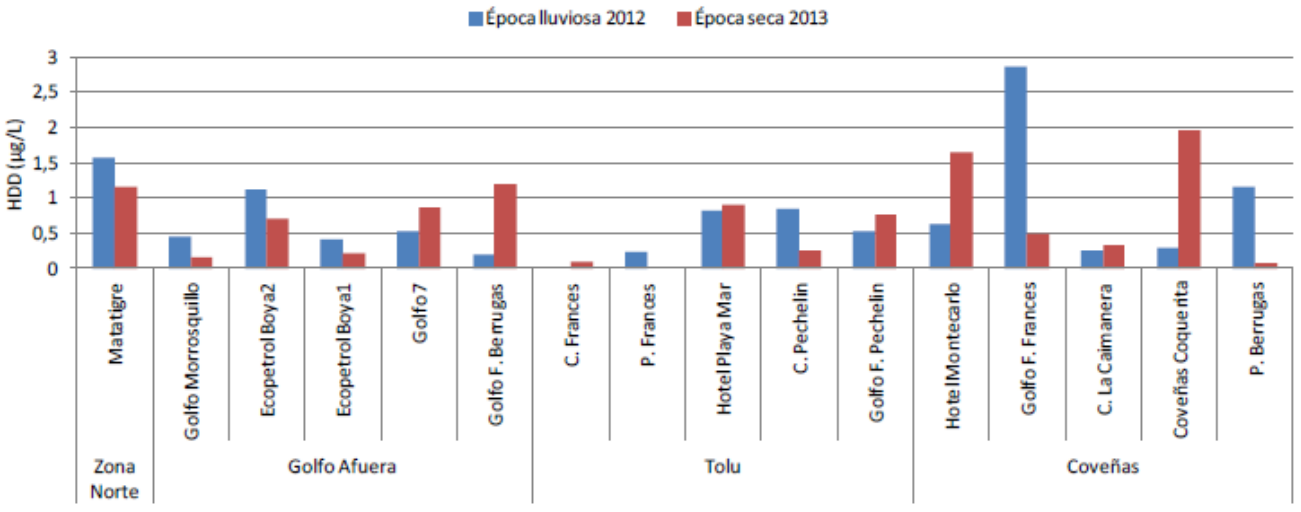

Figura 11. Concentraciones de hidrocarburos disueltos y dispersos ( $\mu \mathrm{g} / \mathrm{L}$ ) medidas en estaciones de aguas superficiales de Sucre en la época lluviosa 2012 y la época seca 2013

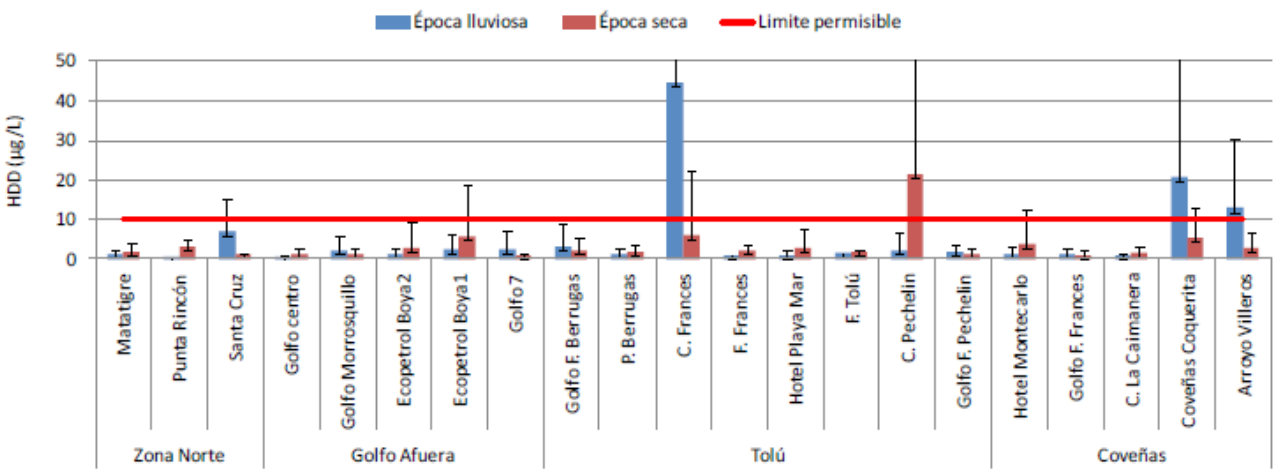

Figura 12. Concentraciones de hidrocarburos disueltos y dispersos ( $\mu \mathrm{g} / \mathrm{L})$ medidas en estaciones de aguas superficiales de Sucre en la época lluviosa 2012 y la época seca 2013 
En lo concerniente a la contaminación de las aguas subterráneas, es un hecho que la incidencia de la característica climática del área de estudio, representada por la evapotranspiración real, sumada al cambio en el uso y manejo de los suelos y de la vegetación establece los factores que limitan, demasiado, la disponibilidad de agua para la recarga neta del acuífero. A ello contribuye, aunque en menor grado, la baja conductividad hidráulica de las zonas, no saturada y saturada, aunque en la primera de ellas es una fortaleza para la atenuación de los contaminantes provenientes de la superficie del suelo (GUTIERREZ, 2004).

Todo lo anotado anteriormente, en el marco de la predicción cualitativa de los impactos ambientales, significa que la variación de las reservas de agua en el acuífero, en época de sequía, es de carácter negativo, es decir, significa un agotamiento anual de las aguas dulces almacenadas de modo permanente, aunque a baja tasa. Este agotamiento físicamente se traduce en la alteración de la calidad de agua, y es un proceso dinámico de Salinización, de frecuencia anual. Cada año hay una renovación de agua dulce por agua de mar que intrusiona ó invade el acuifero (GUTIERREZ, 2004).

La aplicación de la técnica DRASTIC como herramienta de predicción especifica de los impactos ambientales en el acuífero Golfo de Morrosquillo, por causa de la salinización, condujo a la determinación de la vulnerabilidad del recurso hídrico en explotación, en las categorías alta y moderadamente alta. Al analizar los cálculos de éste resultado, se observa que los factores topografía y el tipo de medio acuífero son los que propician mayormente el carácter vulnerable en grado alto.

Esto significa que las características naturales del aluvión fluvio-marino, como son la geomorfología (una llanura de pendiente muy suave hacia la línea de costa) y el tipo de rocas sedimentarias en explotación dan lugar a un mayor tiempo de contacto del agua dulce del embalse subterráneo con el agua contaminante infiltrada, parte de la cual pércola como recarga neta (GUTIERREZ, 2004).

Con el fin de establecer una correlación con los resultados obtenidos mediante la técnica Drastic, como aporte al análisis de vulnerabilidad se aplica también la metodología AVI (Aquifer Vulnerability Index). Es un procedimiento simplificado que cuantifica la vulnerabilidad a través de un parámetro denominado Resistencia Hidráulica C, la que corresponde a una estimación del tiempo de viaje del contaminante a través de la zona saturada, en años (VAN STEMPROORT 1992. Apud. GONZÁLEZ, 2002).

El cálculo del tiempo de viaje se realiza empleando la expresión:
En donde $d_{i}$ corresponde al espesor de capas homogéneas ubicadas sobre el sistema saturado mientras que $\mathrm{K}_{\mathrm{i}}$ es la permeabilidad ó conductividad hidráulica asociada al suelo. Según los valores del tiempo de viaje y el valor del logaritmo decimal de C (log C) se estima la vulnerabilidad (Tabla 3) (GUTIERREZ, 2004), así:

Tabla 3. Resistencia Hidráulica C vs. Vulnerabilidad AVI

\begin{tabular}{ccc}
\hline $\begin{array}{c}\text { Resistencia Hidráulica } \\
\text { C (años) }\end{array}$ & Log ( C ) & Vulnerabilidad AVI \\
\hline$<10$ & $<1$ & Extremadamente alta \\
$10-100$ & 1 a 2 & Muy Alta \\
$100-1000$ & 2 a 3 & Moderadamente Alta \\
$1000-10000$ & 3 a 4 & Baja \\
$>10000$ & $>4$ & Extremadamente Baja \\
\hline
\end{tabular}

Con base en la información litológica ó de las columnas estratigráficas características del área de estudio, en el área urbana y en el campo de pozos municipal de Santiago de Tolú, según trabajos de HERRERA et al.(1997) (Apud. GUTIERREZ (2004) el tiempo de viaje ó resistencia hidráulica, en años, tiene valores en el rango de 0,21 a 2,74 (2,5 a 33 meses) y para todos los casos analizados la vulnerabilidad califica como extremadamente alta. El análisis se hizo para todos los pozos en explotación (5) más 2 pozos del sistema de acueducto, hace dos años (Palo Hueco y Tres Palmas); los espesores de las capas homogéneas pertinentes van desde 18.3 metros en Palo Hueco a 65,5 m en Tres Palmas., con información estratigráfica para aplicar la técnica $\mathrm{AVI}$ ).

De acuerdo al Nacional Academy Council (1993) el concepto de Vulnerabilidad del agua subterránea se refiere a la tendencia ó probabilidad de que un contaminante alcance una posición especificada en el sistema acuífero, después de su introducción en algún punto sobre el terreno (GONZALEZ, 2002).

El análisis de los resultados de vulnerabilidad indica que el acuífero golfo de Morrosquillo reunía las condiciones para ser intrusionado por el mar periódicamente y su tendencia actual es la salinización total de sus aguas, y perdería de modo irreversible la calidad de fuente de abastecimiento de agua dulce aceptable para el consumo humano directo ó en su estado natural (GUTIERREZ, 2004).

\section{Conclusiones}

En las estaciones de la zona Norte se continúan registrando temperaturas cercanas a $30^{\circ} \mathrm{C}$, lo cual implica un riesgo de degradación de los corales por blanqueamiento durante ambas épocas climáticas. 
Los valores de $\mathrm{pH}$, salinidad y oxígeno disuelto se encontraron dentro de los rangos de variación histórica del Departamento, sin embargo, según los resultados actuales y los reportes históricos, estaciones estuarinas y fluviales como caño Guacamayo, caño Alegría y caño Francés han presentado en época seca condiciones de oxigenación por debajo del valor minino permisible establecido por la legislación Colombiana para efectos de uso para la preservación de la fauna y flora.

La medición de coliformes termotolerantes en las playas no evidenció diferencias entre épocas climáticas pero si fue recurrente el detrimento de la calidad de las aguas de Coveñas Coquerita, lo cual representa un riesgo para el desarrollo de actividades como la natación y buceo.

Las concentraciones de hidrocarburos HDD en aguas son inferiores al valor de referencia $(<10 \mu \mathrm{g} / \mathrm{L})$ para aguas no contaminadas. La tendencia general en las concentraciones de hidrocarburos observada es de decrecimiento, a excepción de los casos puntuales en caño Pechelin, caño Francés, Coveñas Coquerita y arroyo Villeros, ocasionado por manejo de crudo y sus derivados, operaciones de transporte y actividades marítimas propias de la región.

Las aguas subterráneas del acuífero son contaminadas por los residuos líquidos y lixiviaciones de residuos sólidos que se infiltran desde las áreas superficiales del techo de su basamento, y las aguas marinas del Golfo, contaminadas por patógenos, hidrocarburos y sólidos suspendidos totales, invaden las capas freáticas, coadyuvando, entre otras, al aumento del gradiente de salinización de tan importante recurso hídrico, único y fundamental para el abastecimiento a la Subregión Morrosquillo.

\section{Referencias}

GESAMP. 2001. A Sea of Troubles. Reports and Studies No. 70. Joint Group of Experts on the Scientific Aspects of Marine Environmental Protection. Publisher UNEP Edit.2001. London, England.

GONZÁLEZ, L. 2002. Vulnerabilidad de Los Acuíferos Semiconfinados Multicapas en el Valle Central, Centro-Sur, Hidrored. Universidad de Chile, Santiago de Chile, Chile.

GUTIÉRREZ, G. 2004 Diagnóstico Ambiental del Acuífero Costero Morrosquillo Zona litoral Tolú-Coveñas. Tesis Maestría. Universidad Nacional de Colombia, Bogotá, Colombia.

INGEOMINAS. 2002. Servicio Geológico Minero (SGC). Mapa Hidrogeológico de Sucre. Bogotá, Colombia. Disponible en: www.icde.org.co/web/guest/ingeominas. Consultado: 10-08-2014.

INVEMAR. 2013. Diagnóstico y Evaluación de la Calidad de las Aguas Marinas y Costeras del Caribe y Pacifico

Colombianos. Instituto de Investigaciones Marinas y Costeras. Santa Martha, Colombia.

MAAMA. 2012. Viñas, Lucía. Instituto Español de Oceanografía. Estrategias Marinas: Evaluación Inicial, Buen Estado Ambiental y Objetivos Ambientales. Ministerio de Agricultura, Alimentación y Medio Ambiente. Madrid, España.

MADS. 2000. Reglamento Técnico de Agua Potable y Saneamiento Básico,

RAS 2000. Ministerio de Medio Ambiente y Desarrollo Sostenible. Bogotá, Colombia.

MADS. 2007. Sistema de Información del Recurso Hídrico Marino SIRHM. Decreto 1353/2007, Bogotá, Colombia.

MINSALUD. 1984. Decreto Ley No. 1594/84 Vigente. Control de Vertimientos Líquidos a Cuerpos de Aguas. Bogotá, Colombia.

MONTESINO, O. 2014. Vulnerabilidad a la Salinización y Perímetro de Protección de Pozos en el Acuífero Costero Morrosquillo, Municipio de Santiago de Tolú, Sucre, Colombia. Universidad de Sucre, Sincelejo, Colombia. 
ONGLEY, E. 1997 Lucha Contra la Contaminación Agrícola de los Recursos Hídricos. Disponible en: http://www. fao.org/docrep/W2598S/W2598S00.htm. Consultado:09-04-2014.

PEREZ, D. 1995 Explotación de Aguas Subterráneas. Un Nuevo Enfoque. Publisher - Científica Técnica. ISPJAE. Marianao, Cuba.

REDCAM. 2013. Programa de Monitoreo de la Calidad de las Aguas Marinas y Costeras de Colombia. REDCAM. Invemar. Santa Marta, Colombia.

SÁNCHEZ, J. 2009. Depto. de Geología. Universidad de Salamanca, Salamanca, España. Disponible: http://web. usal.es/javisan/hidro/bibliografia.htm. Consultado: 10-08-2014. 